\title{
Recent development on the synthesis techniques and properties of graphene derivatives
}

\begin{abstract}
Graphene, an atomically thin two-dimensional carbonaceous nanomaterial, has attracted tremendous research interest in both scientific studies and technological development due to its exceptional electric, mechanical, and chemical properties. Since the discovery of graphene, many efforts have been done to modify the graphene structure for integrating this promising material to vast applications. Specific attention is devoted to the recent progresses on the development of graphene derivatives such as graphene oxide, porous graphene/graphene oxide, reduced graphene oxide, and graphene quantum dots. In this chapter, the definition, intrinsic properties, and various approaches for the synthesis of graphene derivatives based on top-down and bottom-up approaches are discussed. Furthermore, the related works on the preparation of graphene derivatives via chemical oxidation method are also included. In addition, the pitted and peeled out mechanism of the formation of graphene derivatives was also highlighted, and this will lead to a better understanding of the physicochemical properties of graphene derivatives.
\end{abstract}

Keyword: Graphene oxide; Nanographene oxide; Graphene oxide quantum dot; Chemical oxidation; Graphite nanofibers 